\title{
A New Gases Identifying Method With MOX Gas Sensors Using Noise Spectroscopy
}

\author{
Sami Gomri, Thierry Contaret, and Jean-Luc Seguin
}

\begin{abstract}
Gas sensing techniques are often required to not only be sensitive and portable but also to be able to identify gases. In this paper, we describe and demonstrate a new gas identification approach based on noise spectroscopy. By using the model of the gas sensor noise developed in our previous work, we calculate the exact theoretical expression of the first derivative of the power spectral density of the gas sensor noise, and we show that there is a correlation between this expression and the nature of the detected gas. This theoretical result is argued by some experimental results performed on a metal oxide gas sensor exposed to various gases. The new principle holds prospects for finding powerful method for the identification of gases.
\end{abstract}

Index Terms-Electronic nose, gas sensor, noise, spectral analysis.

\section{INTRODUCTION}

$\mathbf{O}$ VER the last decade, "electronic nosing" or "e-nosing" technologies have undergone important developments from a technical and commercial point of view. The expression "electronic nosing" refers to the capability of reproducing human nose using sensor arrays and pattern recognition systems. Electronic nose is a sophisticated system used in the identification of complex mixtures of chemicals. It consists of a multi sensor array, an information-processing unit, software with digital pattern-recognition algorithms, and referencelibrary databases [1]-[5]. The sensor array is composed of different sensors chosen to respond to a wide range of chemical classes and discriminate diverse mixtures of possible analytes. The output of individual sensors are collectively assembled and integrated to generate a distinct digital response pattern. Identification and classification of an analyte mixture is accomplished through recognition of this unique chemical signature (electronic fingerprint) of collective sensor responses.

Since a few years ago, fluctuation enhanced sensing (FES) has become an active research area in the field of gas sensors [6]. The FES principle uses the fluctuations of the gas sensors' response as an information source. This experimental technique is based on noise spectroscopy: the measurement and the analysis of the power spectral density (PSD) of the fluctuations measured at the terminals of sensors in the

Manuscript received December 26, 2017; revised June 10, 2018; accepted June 11, 2018. Date of publication June 26, 2018; date of current version July 24, 2018. The associate editor coordinating the review of this paper and approving it for publication was Dr. Gymama Slaughter. (Corresponding author: Sami Gomri.)

S. Gomri is with the Micro Electro Thermal Systems Unit, National School of Engineers of Sfax, Sfax 3038, Tunisia (e-mail: sami.gomri14@gmail.com).

T. Contaret and J.-L. Seguin are with CNRS, IM2NP, Universiteì de Toulon, Aix Marseille University, 13007 Marseille, France. presence of one or more gases. Measuring these fluctuations caused by adsorption-desorption and diffusion noise provides enhanced selectivity and sensitivity. In fact, the FES method has been used in many works [6]-[15] to show that basing on noise spectroscopy one gas sensor can be enough to identify gases. It is based on the product $f . S(f)$, where $f$ is the frequency and $S(f)$ is the PSD of the gas sensor noise. Indeed, from the plot of this product some gas identification parameters have been extracted and proposed as a gas signature. For example, in [14], the average slope of the $f . S(f)$ product, fixed for different decades of frequency $f$, has been proposed as one of the possible constitutive parameters for gas recognition. In [15], the parameter used as a gas signature is the characteristic frequency at which the product $f . S(f)$ reaches its maximum.

In this context, the aim of this paper is to propose a new gases identification method based on noise spectroscopy. This method is based on the first derivative (with respect to the frequency) of the PSD of the gas sensor noise measured in the presence of gases. We show that, instead of using the function $f . S(f)$, the first derivative $\left(S^{\prime}(f)\right)$, of the PSD of the gas sensor noise can be used in order to identify gases. We show that $S^{\prime}(f)$ has a minimum which depends on the nature of the detected gas.

The structure of the paper is as follows; in section 2, we present an overview on the feature selection step and its importance in electronic nose. At the end of this paragraph we introduce our method through which we propose a new gas identifying feature. In section 3 , the proposed method is studied under two aspects: theoretical demonstration and experimental validation.

\section{Overview on the Feature Selection in ELECTRONIC NOSE (E-Nose)}

The electronic nose is an application of pattern recognition system (PRS). The aim of a pattern recognition system is to associate each pattern with one of a number of possible pattern classes (or simply classes). Patterns are typically described in terms of multidimensional data vectors, where each component is called a feature. In the case of the electronic nose, the patterns and the classes are, respectively, the responses of the sensor array to odorants and the odorants being considered. As illustrated in Fig. 1, this process can be split into four sequential stages: signal pre-processing, dimensionality reduction, classification, and validation.

The pre-processing stage is often applied in order to select a number of parameters called features, which are descriptive 


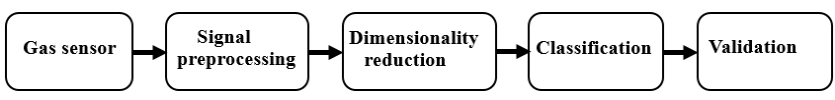

Fig. 1. Generic scheme of a pattern recognition system for electronic nose.

TABLE I

Some Steady-State Features Models [17]-[21]

\begin{tabular}{ll}
\hline \hline \multicolumn{1}{c}{ Model } & \multicolumn{1}{c}{ Description } \\
\hline Difference & $x_{i j}^{\text {max }}-x_{i j}^{\text {min }}$ \\
Relative difference & $x_{i j}^{\text {max }} / x_{i j}^{\text {min }}$ \\
Fractional difference & $\left(x_{i j}^{\max }-x_{i j}^{\text {min }}\right) / x_{i j}^{\text {min }}$ \\
Logarithm difference & $\log \left(x_{i j}^{\text {max }} / x_{i j}^{\text {min }}\right)$ \\
\hline
\end{tabular}

Vertical lines are optional in tables. Statements that serve as captions for the entire table do not need footnote letters.

${ }^{\mathrm{a}}$ Gaussian units are the same as cg emu for magnetostatics; $\mathrm{Mx}=$ maxwell, $\mathrm{G}=$ gauss, $\mathrm{Oe}=$ oersted; $\mathrm{Wb}=$ weber, $\mathrm{V}=$ volt, $\mathrm{s}=$ second, $\mathrm{T}=$ tesla, $\mathrm{m}=$ meter, $\mathrm{A}=$ ampere, $\mathrm{J}=$ joule, $\mathrm{kg}=$ kilogram, $\mathrm{H}=$ henry.

of the sensor array response. Features are those measurements which are extracted from a pattern to represent it in the features space. In other words, pattern analysis enables us to use some features to describe and represent it instead of using the pattern itself. Also called characteristics, attributes or signatures, the recognition efficiency and reliability are dependent on their choice [16].

The first feature extraction method is to extract piecemeal signal features from the original temporal response curves of sensors, including steady-state response and transient responses. In the early stages of the published history of E-noses, a variety of steady-state models (maximum value) have been used as feature extraction method for gas sensor signals, as illustrated in Table 1.

Besides the steady-state features of response curves, various other transient features such as derivatives and integrals of original response curves were taken into research in many special applications [21]-[23]. Moreover, in a recent paper [24], we have used two transient parameters in order to enhance the classification performance of a $\mathrm{WO}_{3}$ gas sensor based pattern recognition system. These parameters are the first derivative and the integral of the temporal response $R(t)$ of the gas sensor.

All these features are used if the signal on which is based the electronic nose is the temporal response $R(t)$ of the gas sensor (Fig. 2). However, in case of noise spectroscopybased electronic nose, the useful signal is no longer the temporal response of the gas sensor; it is the fluctuations of the gas sensor response called gas sensor noise (noted by $\delta R(t)$ in Fig. 2) which is superposed with the temporal response $R(t)$. Hence, all the features used in our previous work or cited in literature are no longer valuable. Thus, in this study we propose to draw inspiration from these last transient
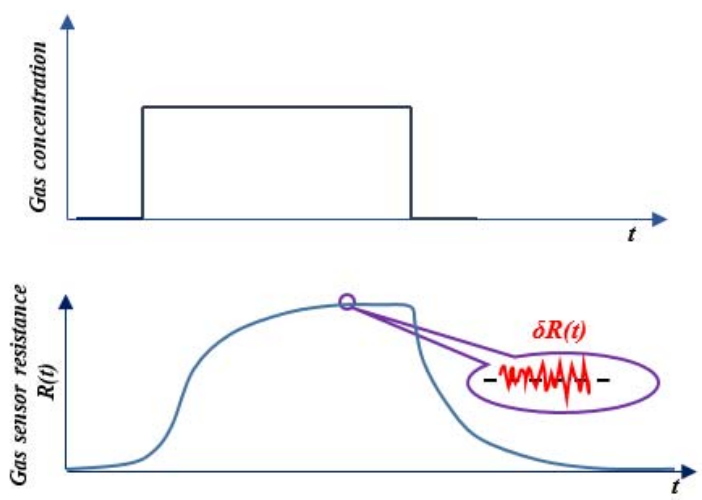

Fig. 2. Typical response $R(t)$ of a MOX gas sensor and its fluctuations $\delta R(t)$.

features by defining new feature which is calculated on the basis of the PSD of the gas sensor noise noted by $S(f)$.

The proposed feature will be the first derivative of the power spectral density (PSD) of the gas sensor noise defined by:

$$
S_{\text {noise }}^{\prime}(f)=\frac{d S_{\text {noise }}(f)}{d f}
$$

where $S_{\text {noise }}(f)$ is the PSD of the gas sensor noise.

In this study we propose to calculate the theoretical expression of the first derivate of the PSD of the gas sensor noise, to seek if there is a correlation between this expression and the nature of the detected gas, and to compare the obtained theoretical result with experimental results.

\section{TheORETICAL ANALYSis}

In our previous work [25], we have developed a model of noise in metallic-oxide gas micro sensors. We have shown that the adsorption-desorption (A-D) noise generated in the overall sensing layer is a combination of multi-Lorentzian components. The number of Lorentzians is independent of the nature of the detected gas. The parameters of each Lorentzian depend on the nature of the detected gas. Moreover, we have shown that at low frequencies the A-D noise will dominate and at high frequencies its will be dominated by a white noise due the thermal noise induced by the sensing layer resistance. We have shown that the plot of the frequency dependency of the PSD of the gas sensor noise have the shape given in Fig. 3.

The calculation of the PSD of the gas sensor noise done in [25] has resulted in the following expression:

$$
S_{\text {noise }}(f)=\sum_{i=1}^{g} L_{i}(f)+S_{\text {therm }}
$$

Where

$$
L_{i}(f)=\frac{H_{i}}{1+\left(\frac{f}{f_{c i}}\right)^{2}}
$$

and

$$
S_{\text {therm }}=\frac{4 k T}{R_{\text {sensor }}}
$$




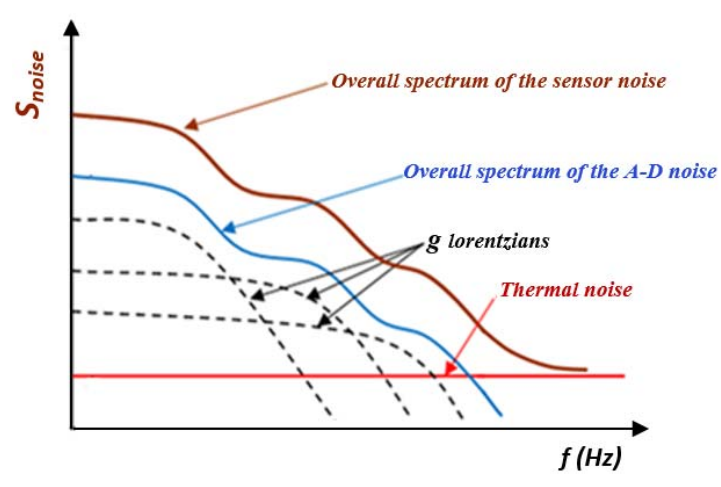

Fig. 3. Decomposition of the overall chemisorption-induced adsorptiondesorption (A-D) noise spectrum into three Lorentzians and contribution of the thermal noise to the overall spectrum of the sensor noise [25].

where $R_{\text {sensor }}$ is the sensing layer resistance. Each parameter $H_{i}$ and $f_{c i}$ are respectively the noise level and cut-off frequency of the Lorentzian number $i$, both having expressions which depend on the nature of the detected gas. $T$ is the temperature and $k$ is the Boltzman constant. $g$ is the number of different grain size involved in the sensing layer microstructure.

On the basis of our previous work [25], we propose, in this section, to calculate the theoretical expression of the first derivative of the PSD of the gas sensor noise, and to show that it admits a minimum which depends on the nature of the detected gas.

Using "(1)", we express the first derivative of the PSD of the gas sensor noise by:

$$
S_{\text {noise }}^{\prime}(f)=\frac{d S_{\text {noise }}(f)}{d f}=\sum_{i=1}^{g} \frac{d L_{i}(f)}{d f}+0=\sum_{i=1}^{g} L_{i}^{\prime}(f)
$$

where

$$
L_{i}^{\prime}(f)=\frac{d L_{i}(f)}{d f}=-2 \frac{H_{i}}{f_{c i}^{2}} \frac{f}{\left[1+\frac{f^{2}}{f_{c i}^{2}}\right]^{2}}
$$

Thus, we obtain the theoretical expression of the first derivative of the PSD of the gas sensor noise:

$$
S_{\text {noise }}^{\prime}(f)=-2 \sum_{i=1}^{g} \frac{H_{i}}{f_{c i}^{2}} \frac{f}{\left[1+\frac{f^{2}}{f_{c i}^{2}}\right]^{2}}
$$

From "(7)", we can conclude that, in order to plot the curve of the first derivative of PSD of the sensor noise, it is more convenient to begin by considering the first derivative of a single Lorentzian $L_{i}^{\prime}(f)$. Then, once we obtain the plot of one first derivative $L_{i}^{\prime}(f)$, we can easily deduce the plot of the first derivative of the whole PSD of the sensor noise.

In order to plot the frequency dependence of the first derivative of a single Lorentzian $L_{i}^{\prime}(f)$ we should better make a mathematical study of the function $L_{i}^{\prime}(f)$ for positive frequencies. This mathematical study can be done by calculating the first derivative of $L_{i}^{\prime}(f)$ (that means the second derivative of $\left.L_{i}(f)\right)$ and studying its sign for positive frequencies.

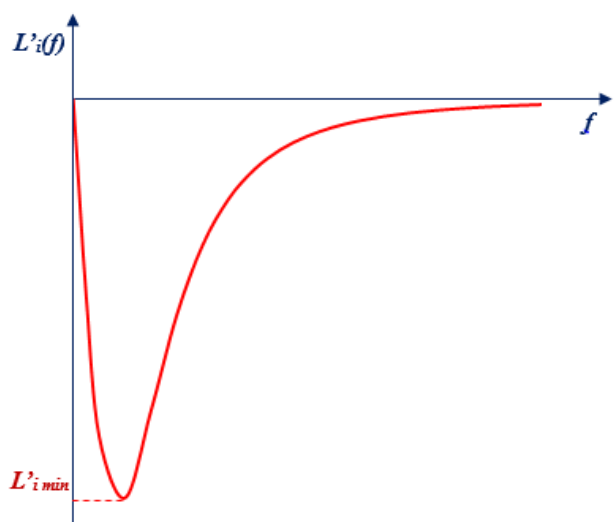

Fig. 4. Plot of the first derivative of $L_{i}^{\prime}(f)\left(H_{i}\right.$ and $f_{c i}$ are chosen arbitrary (respectively 16 and 8 ) to show the shape of the plot).

The calculation of the second derivative of $L_{i}^{\prime}(f)$ gives:

$$
L_{i}^{\prime \prime}(f)=-2 \frac{H_{i}}{f_{c i}^{2}} \frac{1-3 \frac{f^{2}}{f_{c i}^{2}}}{\left[1+\frac{f^{2}}{f_{c i}^{2}}\right]^{3}}
$$

Using "(6)", we can easily show that $L_{i}^{\prime}(f)$ has a minimum at the frequency $f_{c i} / \sqrt{3}$ and this minimum is:

$$
L_{i \min }^{\prime}=L_{i}^{\prime}\left(\frac{f_{c i}}{\sqrt{3}}\right)=-\frac{9}{8 \sqrt{3}} \frac{H_{i}}{f_{c i}}
$$

By studying the sign of the expression given in "(6)", we obtain the plot of the function $L_{i}^{\prime}(f)$ (see Fig. 4).

Hence, the first derivative of each lorentzian $L_{i}^{\prime}(f)$ has a minimum (whose expression is given by "(9)") at the frequency $f_{c i} / \sqrt{3}$.

Now, let's extend our mathematical analysis to the first derivative of the whole PSD of the gas sensor noise $S_{\text {noise }}^{\prime}(f)$. Equation (5) shows that the first derivative of the PSD of the gas sensor noise $\left(S_{\text {noise }}^{\prime}(f)\right)$ is a summation of multi functions $\left(L_{i}^{\prime}(f)\right)_{1<i<g}$. Besides, we have just shown that each function $L_{i}^{\prime}(f)$ has a minimum $L_{i m i n}^{\prime}$ at a frequency $f_{c i} / \sqrt{3}$. Thus, we can easily show that the function $S_{\text {noise }}^{\prime}(f)$ has also a minimum $S_{0}^{\prime}$ at a frequency $f_{0}$ which satisfies (see Appendix):

$$
\left\{\begin{array}{l}
\frac{f_{c 1}}{\sqrt{3}}<f_{0}<\frac{f_{c g}}{\sqrt{3}} \\
S_{0}^{\prime}=S_{\text {noise }}^{\prime}\left(f_{0}\right)
\end{array}\right.
$$

In Fig. 5, we show an example of a plot of the first derivative of the PSD of the gas sensor noise considering a combination of four functions $\left(L_{i}^{\prime}(f)\right)_{1<i<4}$

Moreover, from "(4)" and "(5)", the first derivative of the PSD of the gas sensor noise $S_{\text {noise }}^{\prime}(f)$ can be mathematically considered as a parametric function, which has $2 g$ parameters. These parameters are the various noise levels $\left(H_{i}\right)_{1<i<g}$ and cut off frequencies $\left(f_{c i}\right)_{1<i<g}$ which are involved in the expression of the PSD of the gas sensor noise (see "(2)" and "(3)"). Bearing in mind that all these parameters depend on the nature of the detected gas (see [25]), it is clear that the output of each input by this function depends on the nature of the detected gas. Using "(9)", we can easily see that the 


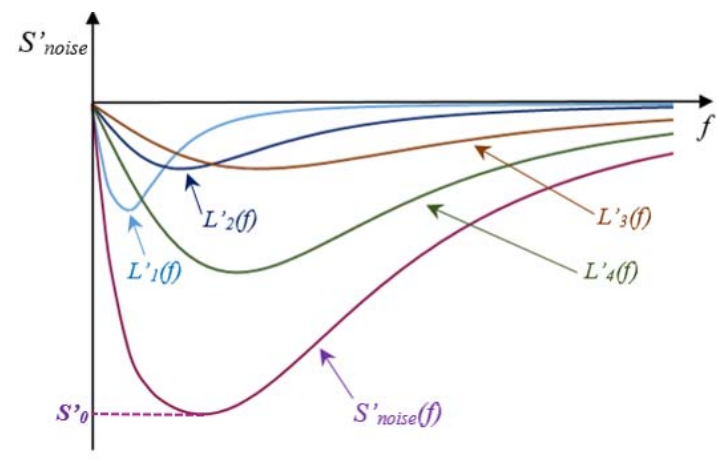

Fig. 5. An example of a simulation of the first derivative of the PSD of the gas sensor noise.

minimum $S_{0}^{\prime}$ of the first derivative $S_{\text {noise }}^{\prime}(f)$ is the output of $f_{0}$ by the function $S_{\text {noise }}^{\prime}(f)$; it expresses by:

$$
S_{0}^{\prime}=S_{\text {noise }}^{\prime}\left(f_{0}\right)=-2 \sum_{i=1}^{g} \frac{H_{i}}{f_{c i}^{2}} \frac{f_{0}}{\left[1+\frac{f_{0}^{2}}{f_{c i}^{2}}\right]^{2}}
$$

Finally, we have shown that the first derivative of the PSD of the gas sensor noise has a minimum which depends on the nature of the detected gas. This theoretical result will be compared with experimental results in the following section.

\section{EXPERIMENTAL VALIDATION}

In order to check the consistency of this gas identification method, we will apply it to some experimental results by plotting the frequency dependency of the first derivative $\left(S_{\text {noise-meas }}^{\prime}(f)\right)$ of the PSD of the measured gas sensor noise $\left(S_{\text {noise-meas }}(f)\right)$ for different gases and comparing it to the theoretical analysis shown in section 3.1. However, before that, we have to check the consistency of the theoretical model of the gas sensor noise (given by "(2)") on which our new gas identification method is based. Hence, in order to plot $S_{\text {noise-meas }}^{\prime}(f)$, we have to measure the gas sensor noise $S_{\text {noise-meas }}(f)$ under different gases and compare the obtained experimental noise spectra to the theoretical model. After that, we calculate (using the ORIGIN software) the first derivative of the measured gas sensor noise spectra $S_{\text {noise-meas }}^{\prime}(f)$, and we plot it for different gases.

\section{A. Noise Responses Under Different Gases}

Experimental noise measurements have been performed on metal-oxide gas microsensors with $\mathrm{WO}_{3}$ sensing layer. $\mathrm{WO}_{3}$ thin films were prepared by reactive radio frequency (13.56 MHz) magnetron sputtering, using a $99.9 \%$ pure tungsten target. The films were sputtered on $\mathrm{SiO}_{2} / \mathrm{Si}$ substrates with platinum electrodes, in a reactive atmosphere of oxygen-argon mixture.

The experimental setup, which we used to measure the sensor noise response in various gases, is presented in Fig. 6. The power spectrum of the amplified current fluctuations across the sensor is measured in the frequency range of $0.1 \mathrm{~Hz}$ - $100 \mathrm{kHz}$ using low noise current preamplifier. The sensors are placed in a stainless steel measurement chamber where mixed gases can be admitted and evacuated via distribution

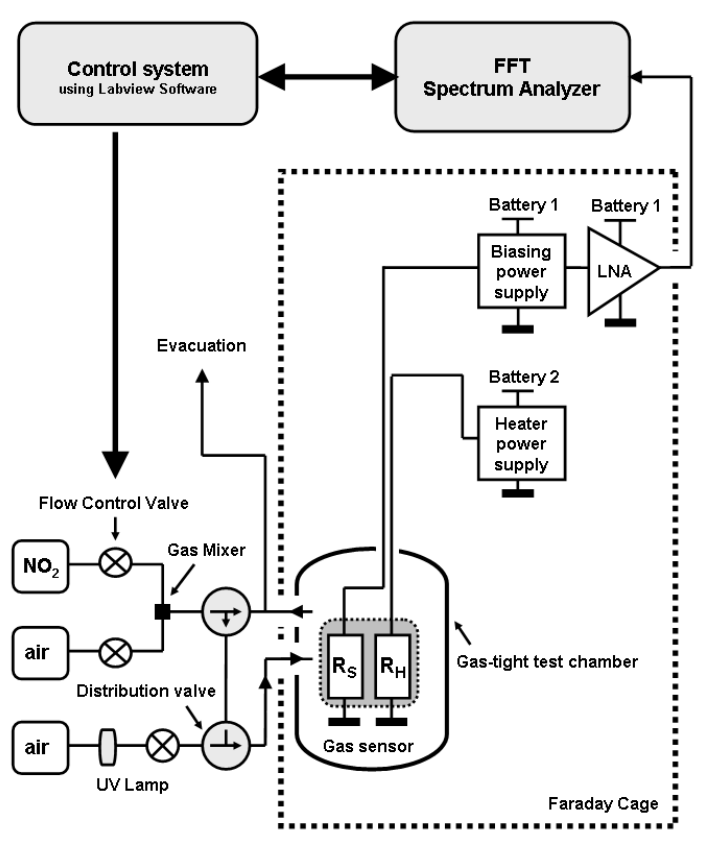

Fig. 6. General Diagram of measurements system used to characterize microsensor noise responses.

valves. The gas chamber, the preamplifier, the sensors biasing and the power supply for sensor local heating are incorporated in a Faraday cage. All these devices are battery powered. For the present study, the gas sensor was exposed to three gases: carbon monoxide, nitrogen dioxide and ozone, diluted in dry air.

In order to quantify the effect of various chemical environments on the resistance of the sensing layer we compare the PSD of the current fluctuations in three chemical species. In Fig. 7 we present the frequency dependency of the PSD of gas sensor current fluctuations measured when the gas sensor is exposed to: carbon monoxide, ozone and nitrogen dioxide.

Comparing the gas sensor's noise measurements, given in Fig. 6, with the theoretical spectrum obtained in our previous paper (see Fig. 3), we can see clearly the consistency of our previous theoretical model. Indeed, from the measured gas sensor noise presented in Fig. 7, we note that for frequencies greater than $100 \mathrm{~Hz}$ all spectra are almost confounded and present a white noise. However below this frequency, the overall spectrum of the gas sensor noise is a combination of multi Lorentzians. This result was shown in our previous paper [25]. Moreover, in the model presented in our previous paper [25], we have shown that the number of lorentzians is independent of the nature of the detected gas. This result is confirmed by the measured gas sensor noise spectra presented in Fig. 5 where we obtained three lorentzians whatever the tested gas.

In table II we present the extracted lorentzians parameters. After summing the three extracted lorentzians, we obtain the modeled plot presented by curve number 2 in Fig. 6 . We note that the curve of the modeling (curve 2) is almost confounded with that of the measure (curve 1). That means the noise generated by the adsorption of either carbon monoxide, 

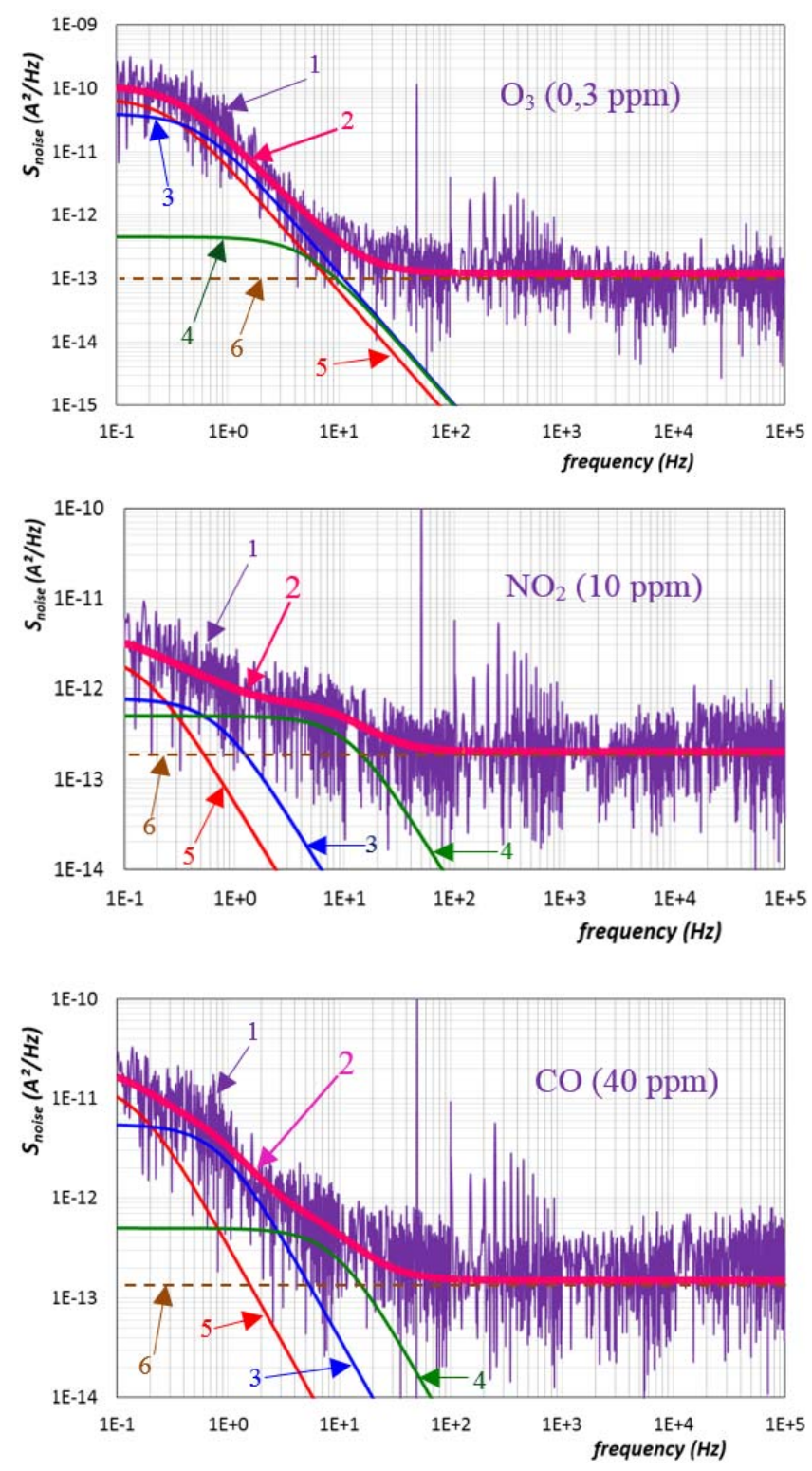

Fig. 7. Decomposition of noise response of the sensor under $0.3 \mathrm{ppm}$ of ozone, 10ppm of nitrogen dioxide and $40 \mathrm{ppm}$ of carbon monoxide in dry air. 1: the measured spectrum, 2 (thick and red) line : fitted spectrum, 3-5: lorentzians constituting the low frequency part of the spectrum "eq.3"; dotted line 6 : thermal noise.

ozone or nitrogen dioxide is described by a decomposition into three Lorantzians. The number of lorentzians is independent of the nature of the detected gas. Using a modeling of all lorentzians (see Fig. 6), we get an extraction of all lorentzians parameters given in table II. From the various values given in table II we note that extracted parameters of each lorentzian involved in the expression of the PSD of the gas sensor noise depend on the nature of the detected gas. These results show the consistency of the theoretical model of the PSD of the gas sensor noise given in our previous paper [25].

\section{B. First Derivative of the PSD of the Noise Responses Under Different Gases}

The first derivative $S_{\text {noise-meas }}^{\prime}(f)$ of the PSD of the measured gas sensor noise is calculated using the ORIGIN
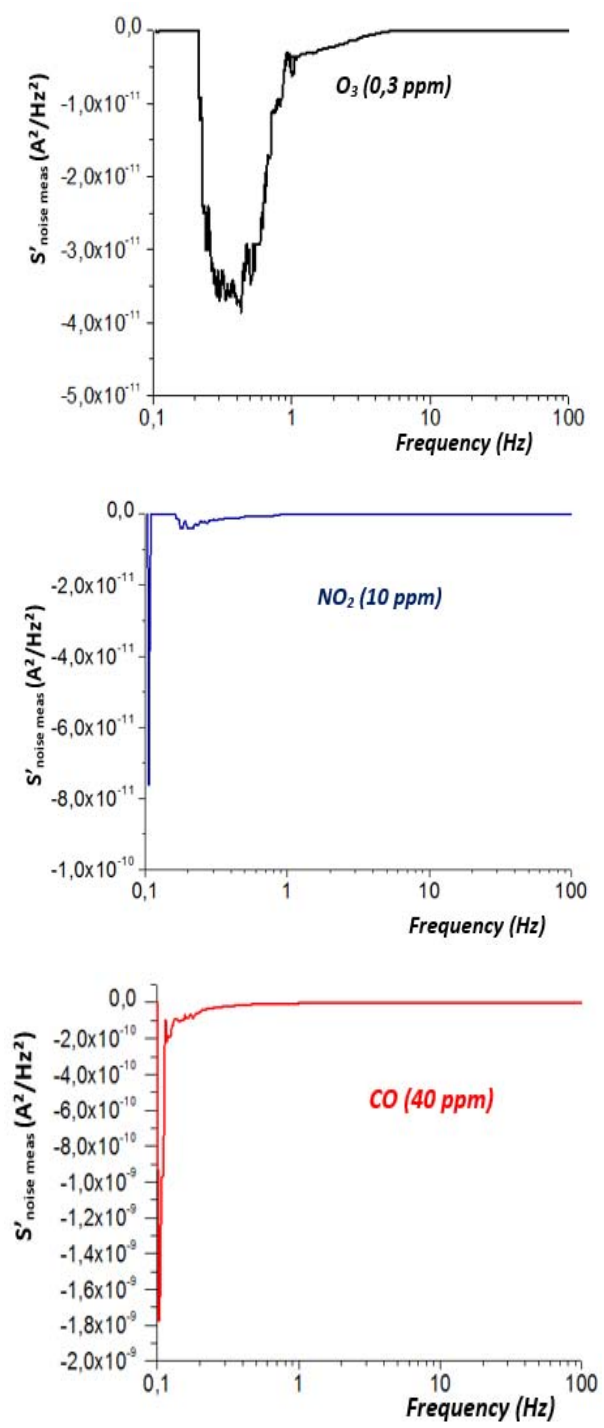

Fig. 8. Plots of the first derivative of the PSD of the gas sensor noise under carbon monoxide, ozone and nitrogen dioxide.

TABLE II

Extracted Noise Parameters

\begin{tabular}{llll}
\hline \hline \multicolumn{1}{c}{ Gases } & \multicolumn{1}{c}{$\mathrm{O}_{3}$} & $\mathrm{CO}$ & \multicolumn{1}{c}{$\mathrm{NO}_{2}$} \\
\hline$H_{1}\left(A^{2} / H z\right)$ & $7 \cdot 10^{-11}$ & $2.10^{-12}$ & $1,5 \cdot 10^{-11}$ \\
$f_{c l}(H z)$ & 0,3 & 0,25 & 0,2 \\
$H_{2}\left(A^{2} / H z\right)$ & $4.10^{-11}$ & $5,5 \cdot 10^{-13}$ & $7.10^{-12}$ \\
$f_{c 2}(H z$ & 0,55 & 0,7 & 0,85 \\
$H_{3}\left(A^{2} / H z\right)$ & $4,53 \cdot 10^{-13}$ & $6.10^{-13}$ & $5.10^{-13}$ \\
$f_{c 3}(H z)$ & 5 & 11 & 9,5 \\
$S_{t h}\left(A^{2} / H z\right)$ & $1,2 \cdot 10^{-13}$ & $1,5 \cdot 10^{-13}$ & $2.10^{-13}$
\end{tabular}

software. In Fig. 8 we present the plots of the first derivative of the PSD of the gas sensor noise response under carbon monoxide, ozone and nitrogen dioxide. From these plots we can see clearly that either in case of carbon monoxide, ozone or nitrogen dioxide, the first derivative of the measured gas sensor noise spectrum present a negative minimum $S^{\prime}{ }_{0}$ Exp which is in consistency with our theoretical result. Moreover, from the values of $\mathrm{S}^{\prime}$ oexp shown in Table III, we note that each 
TABLE III

Minimum of The First Derivative of THE PSD OF THE GAS SENSOR NOISE

\begin{tabular}{cccc}
\hline \hline Gases & $\mathrm{O}_{3}$ & $\mathrm{NO}_{2}$ & $\mathrm{CO}$ \\
\hline$S_{\text {O exp }}^{\prime}$ & $-3,9 \cdot 10^{-11}$ & $-7,7 \cdot 10^{-11}$ & $-1,77 \cdot 10^{-9}$
\end{tabular}

gas has a specific value of the minimum of the first derivative of the PSD of the gas sensor noise. However, a similarity between the values of $\mathrm{S}{ }_{\text {oexp }}$ obtained in case of $\mathrm{NO}_{2}$ and $\mathrm{O}_{3}$ can be easily noted. In fact, from "(11)", we can see clearly that the minimum of the PSD of the gas sensor noise depends on two parameters: $H_{i}$ and $f_{c i}$. Indeed, these parameters are expressed in [25], where we have shown that they depend on the nature of the detected gas by means of the molecule mass and size, and its adsorption energy. Thus, the similarity between the values of $\mathrm{S}^{\prime}{ }_{0}$ exp obtained in case of $\mathrm{NO}_{2}$ and $\mathrm{O}_{3}$ can be explained by the similarity between $\mathrm{O}_{3}$ and $\mathrm{NO}_{2}$ molecules. Indeed, the two molecules differ only by one atom $(\mathrm{O}$ replaced by $\mathrm{N}$ ), and these atoms are very close in mass (atomic weight: 16 and 14 respectively) and radius (radius of Van der Waals: $1.55 \AA$ and $1.6 \AA$ respectively [31]).

Such result show the consistency of our gases identification method. Thus, we have shown that the function $S_{\text {noise }}^{\prime}(f)$ has a minimum which depends on the nature of the detected gas. As this minimum is rather sharp, this is a sensitive and accurate method to identify gases.

\section{CONClusion}

In this paper, we have presented a new gases identification method based on noise spectroscopy. It was based on the plot of the frequency dependency of the first derivative of the power spectral density (PSD) of the gas sensor noise. Based on our latest noise modeling results in MOX gas sensors, we have calculatedthe exact expression of the first derivative of the PSD of the gas sensor noise and shown that it has a negative minimum which depends on the nature of the detected gas. Moreover, in order to validate this new theoretical result with some experimental noise measurements, we have performed a new low frequencies noise measurements in metal oxide gas sensor under three different gases: carbon monoxide, ozone, and nitrogen dioxide. Using the obtained noise measurements we have confirmed the consistency of both the theoretical model of the gas sensor noise presented in our previous paper, and the new gases identification method which is presented in this paper. Such a method can be used in order to fingerprint a gas. The capability of this method for discriminating gases can be evaluated using a principal components analysis (PCA technique). This will be done in future work comparing several identification methods based on FES technique.

\section{APPENDIX}

How to show that the function $S_{\text {noise }}^{\prime}(f)$ has a minimum $S_{0}^{\prime}$ and that this minimum is reached at a frequency $f_{0}$ such as $\frac{f_{c 1}}{\sqrt{3}}<f_{0}<\frac{f_{c g}}{\sqrt{3}}$

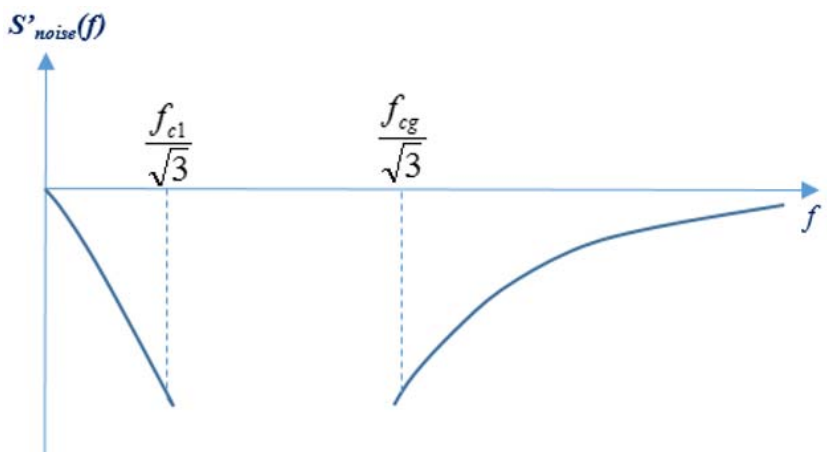

Fig. 9. The first part of the plot of $S_{\text {noise }}^{\prime}(f)$.

The first derivative of the gas sensor noise $S_{\text {noise }}^{\prime}(f)$ is expressed by:

$$
S_{\text {noise }}^{\prime}(f)=\frac{d S_{\text {noise }}(f)}{d f}=\sum_{i=1}^{g} \frac{d L_{i}(f)}{d f}+0=\sum_{i=1}^{g} L_{i}^{\prime}(f)
$$

Where

$$
L_{i}^{\prime}(f)=\frac{d L_{i}(f)}{d f}=-2 \frac{H_{i}}{f_{c i}^{2}} \frac{f}{\left[1+\frac{f^{2}}{f_{c i}^{2}}\right]^{2}}
$$

In order to study $S_{\text {noise }}^{\prime}(f)$, we have to study the sign of its first derivative which means the sign of $S_{\text {noise }}^{\prime \prime}(f)$.

The first derivative of $S_{\text {noise }}(f)$ is:

$$
S^{\prime \prime} \text { noise }(f)=\sum_{i=1}^{g} L_{i}^{\prime \prime}(f)
$$

where

$$
L_{i}^{\prime \prime}(f)=-2 \frac{H_{i}}{f_{c i}^{2}} \frac{1-3 \frac{f^{2}}{f_{c i}^{2}}}{\left[1+\frac{f^{2}}{f_{c i}^{2}}\right]^{3}}
$$

From equation "(A.2)", one can easily see that:

$$
\begin{cases}\text { for each } f<\frac{f_{c i}}{\sqrt{3}} & L_{i}^{\prime \prime}(f)<0 \\ L_{i}^{\prime \prime}\left(\frac{f_{c i}}{\sqrt{3}}\right)=0 & \\ \text { for each } f>\frac{f_{c i}}{\sqrt{3}} ; & L_{i}^{\prime \prime}(f)>0\end{cases}
$$

Let be $\left(f_{c 1}, f_{c 2}, f_{c 3}, \ldots . . . f_{c g}\right)$ the various cut off frequencies involved in the expression of the PSD of the gas sensor noise (see "(2)" and "(3)" in the manuscript), arranged in the following way:

$$
f_{c 1} \leq f_{c 2} \leq f_{c 3} \leq \ldots \ldots \ldots . . \leq f_{c g}
$$




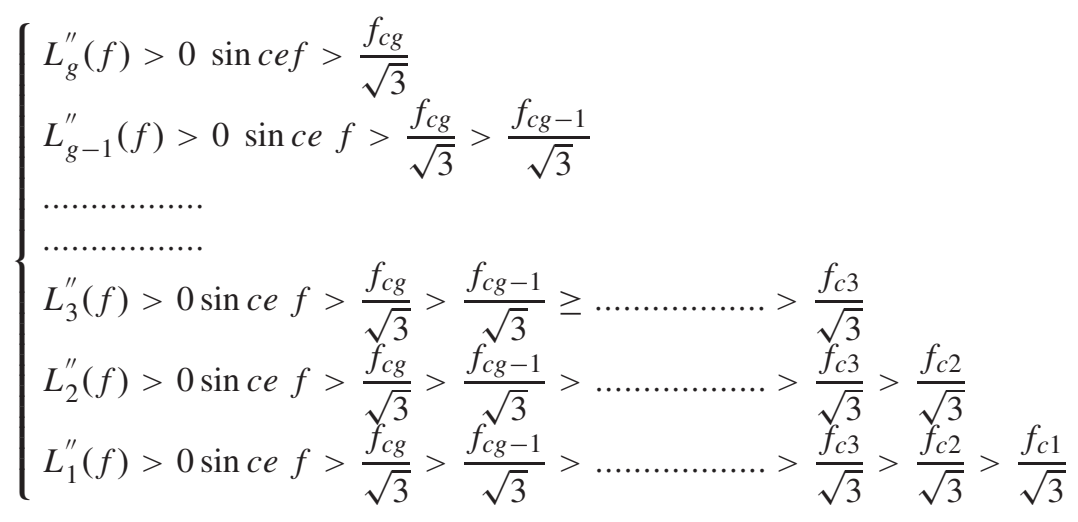

Hence, from "(A.3)" and "(A.4)" we can deduce that for each frequency $f<\frac{f_{c 1}}{\sqrt{3}}$ we have:

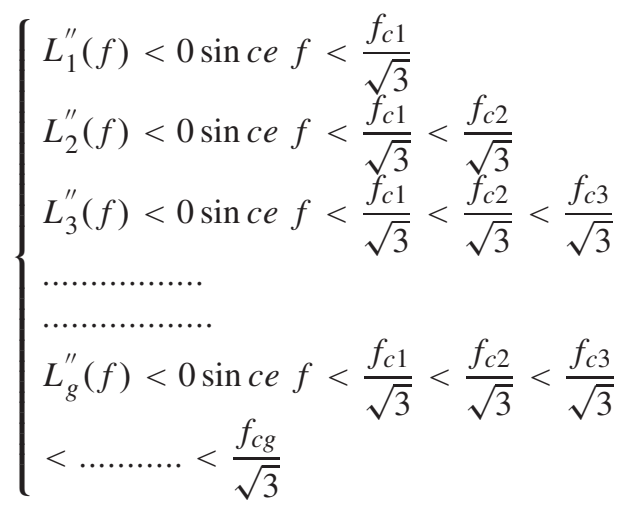

Hence from "(A.1)" and "(A.5)" we deduce that for each $f<$ $\frac{f_{c 1}}{\sqrt{3}}$; we have:

$$
S_{\text {noise }}^{\prime \prime}(f)<0
$$

Now let us study the sign of $S_{\text {noise }}^{\prime \prime}(f)$ when the frequency $f>\frac{f_{c g}}{\sqrt{3}}$.

From "(A.3)" and "(A.4)", we can confirm that for each frequency $f>\frac{f_{c g}}{\sqrt{3}}$ we have (A.9), as shown at the top of this page.

Hence, from "(A.1)" and "(A.7)" we deduce that:

$$
\text { for each } f>\frac{f_{c g}}{\sqrt{3}} ; \quad S_{\text {noise }}^{\prime \prime}(f)>0
$$

Finally, from "(A.7)" and "(A.8)" we conclude that:

$$
\left\{\begin{array}{l}
\text { for each } f<\frac{f_{c 1}}{\sqrt{3}} ; S_{\text {noise }}^{\prime \prime}(f)<0 \\
\text { for each } f>\frac{f_{c g}}{\sqrt{3}} ; S_{\text {noise }}^{\prime \prime}(f)>0
\end{array}\right.
$$

Hence, we can conclude that:

- in the interval $\left[0, \frac{f_{c 1}}{\sqrt{3}}\left[\right.\right.$, the function $S_{\text {noise }}^{\prime}(f)$ is decreasing.

- in the interval $\left[\frac{f_{c g}}{\sqrt{3}},+\infty\left[\right.\right.$, the function $S_{\text {noise }}^{\prime}(f)$ is increasing.

On the other hand, using "(A.2)" we deduce that:

$$
\left\{\begin{array}{l}
S_{\text {noise }}^{\prime}(0)=\sum_{i=1}^{g} L_{i}^{\prime}(0)=0 \\
\lim _{f \rightarrow+\infty}\left[S_{\text {noise }}^{\prime}(f)\right]=0
\end{array}\right.
$$

Hence we obtain the first part of the shape of the curve of the function $S_{\text {noise }}^{\prime}(f)$ (see Fig. 9)

It is worth noting that the function $S_{\text {noise }}^{\prime}(f)$ may have more than one local minimum in this interval, but what is certain is that it possesses an absolute minimum in this interval since the curve decreases in the vicinity of $f_{c 1} / \sqrt{3}$ and increases in the vicinity of $f_{c g} / \sqrt{3}$. Hence we conclude that it exists a frequency $f_{0}$ which satisfies:

$$
\left\{\begin{array}{l}
\frac{f_{c 1}}{\sqrt{3}}<f_{0}<\frac{f_{c g}}{\sqrt{3}} \\
S_{\text {noise }}^{\prime}(f) \geq S_{\text {noise }}^{\prime}\left(f_{0}\right) \text { for each } f \geq 0
\end{array}\right.
$$

The value of $S_{\text {noise }}^{\prime}\left(f_{0}\right)$ is the minimum of $S_{\text {noise }}^{\prime}(f)$, it is noted in the manuscript by $S_{0}^{\prime}$.

\section{ACKNOWLEDGMENT}

This research did not receive any specific grant from funding agencies in the public, commercial, or not-in-profit sectors.

\section{REFERENCES}

[1] J. W. Gardner, "Detection of vapours and odours from a multisensor array using pattern recognition. Part 1. Principal component and cluster analysis," Sens. Actuators B, Chem., vol. 4., pp. 109-115, May 1991.

[2] M. S. Freund and N. S. Lewis, "A chemically diverse conducting polymer-based "electronic nose," Proc. Nat. Acad. Sci. USA, vol. 92, pp. 2652-2656, Mar. 1995.

[3] H. Abe, S. Kanaya, Y. Takahashi, and S.-I. Sasaki, "Extended studies of the automated odor-sensing system based on plural semiconductor gas sensors with computerized pattern recognition techniques," Anal. Chim. Acta, vol. 215, pp. 155-168, 1988.

[4] H. V. Shurmer and J. W. Gardner, "Odour discrimination with an electronic nose," Sens. Actuators B, Chem., vol. 8, pp. 1-11, Apr. 1992.

[5] B. R. Kowalski and C. F. Bender, "Pattern recognition. Powerful approach to interpreting chemical data," J. Amer. Chem. Soc., vol. 94, pp. 5632-5639, Aug. 1972.

[6] L. B. Kish, R. Vajtai, and C. G. Granqvist, "Extracting information from noise spectra of chemical sensors: Single sensor electronic noses and tongues," Sens. Actuators B, Chem., vol. 71, pp. 55-59, Nov. 2000.

[7] F. Gasparyan, H. Khondkaryan, and M. Aleksanyan, "New applications of the noise spectroscopy for hydrogen sensors," J. Mod. Phys., vol. 5, pp. 1662-1669, Oct. 2014.

[8] Ł. Lentka, J. M. Smulko, R. Ionescu, C. G. Granqvist, and L. B. Kish, "Determination of gas mixture components using fluctuation enhanced sensing and the LS-SVM regression algorithm," Metrol. Meas. Syst., vol. 22, no. 3, pp. 341-350, 2015.

[9] J. Smulko, C.-G. Granqvist, and L. B. Kish, "On the statistical analysis of noise in chemical sensors and its application for sensing," Fluctuation Noise Lett., vol. 1, pp. L147-L153, Sep. 2001.

[10] A. K. Vidybida, "Adsorption-desorption noise can be used for improving selectivity," Sens. Actuators A, Phys., vol. 107, pp. 233-237, Nov. 2003. 
[11] G. Schmera, C. Kwan, P. Ajayan, R. Vajtai, and L. B. Kish, "Fluctuationenhanced sensing: Status and perspectives," IEEE Sensors J., vol. 8, no. 6, pp. 714-719, Jun. 2008.

[12] C. Kwan, G. Schmera, J. M. Smulko, L. B. Kish, P. Heszler, and C.-G. Granqvist, "Advanced agent identification with fluctuationenhanced sensing," IEEE Sensors J., vol. 8, no. 6, pp. 706-713, Jun. 2008.

[13] G. Schmera and L. B. Kish, "Fluctuation-enhanced gas sensing by surface acoustic wave devices," Fluctuation Noise Lett., vol. 2, pp. L117-L123, Jun. 2002.

[14] B. Ayhan et al., "Fluctuation enhanced sensing (FES) with a nanostructured, semiconducting metal oxide film for gas detection and classification," Sens. Actuators B, Chem., vol. 188, pp. 651-660, Nov. 2013.

[15] S. Rumyantsev, G. Liu, R. A. Potyrailo, A. A. Balandin, and M. S. Shur, "Selective sensing of individual gases using graphene devices," IEEE Sensors J., vol. 13, no. 8, pp. 2818-2822, Aug. 2013.

[16] M. Pardo and G. Sberveglieri, "Comparing the performance of different features in sensor arrays," Sens. Actuators B, Chem., vol. 123, no. 1, pp. 437-443, 2007.

[17] M. Ohnishi, T. Ishibashi, Y. Kijima, C. Ishimoto, and J. Seto, "A molecular recognition system for odorants incorporating biomimetic gassensitive devices using Langmuir-Blodgett films," Sensors Mater, vol. 4, no. 1, pp. 53-60, 1992.

[18] M. Egashira, Y. Shimizu, and Y. Takao, "Trimethylamine sensor based on semiconductive metal oxides for detection of fish freshness," Sens. Actuators B, Chem., vol. 1, pp. 108-112, Jan. 1990.

[19] T. C. Pearce, J. W. Gardner, S. Friel, P. N. Bartlett, and N. Blair, "Electronic nose for monitoring the flavour of beers," Analyst, vol. 118, no. 4, pp. 371-377, 1993

[20] S. W. Moore, J. W. Gardner, E. L. Hines, W. Göpel, and U. Weimar, "A modified multilayer perceptron model for gas mixture analysis," Sens. Actuators B, Chem., vol. 16, pp. 344-348, Oct. 1993.

[21] C. Distante, M. Leo, P. Siciliano, and K. C. Persaud, "On the study of feature extraction methods for an electronic nose," Sens. Actuators B, Chem., vol. 87, no. 2, pp. 274-288, Dec. 2002.

[22] T. Eklöv, P. Mårtensson, and I. Lundström, "Enhanced selectivity of MOSFET gas sensors by systematical analysis of transient parameters," Anal. Chim. Acta, vol. 353, pp. 291-300, Oct. 1997.

[23] S. Zhang, C. Xie, D. Zeng, Q. Zhang, H. Li, and Z. Bi, "A feature extraction method and a sampling system for fast recognition of flammable liquids with a portable E-nose," Sens. Actuators B, Chem., vol. 124, no. 2 , pp. 437-443, 2007.

[24] R. Faleh, M. Othman, S. Gomri, K. Aguir, and A. Kachouri, "A transient signal extraction method of $\mathrm{WO}_{3}$ gas sensors array to identify polluant gases," IEEE Sensors J., vol. 16, no. 9, pp. 3123-3130, May 2016.

[25] S. Gomri, T. Contaret, J. Seguin, K. Aguir, and M. Masmoudi, "Noise modeling in MOX gas sensors," Fluctuation Noise Lett., vol. 16, no. 2, p. 1750013, 2017.
Sami Gomri was born in Sfax, Tunisia, in 1977. He received the Diploma degree in electrical engineering from the National Engineers School of Sfax (ENIS), University of Sfax, Tunisia, in 2001, the D.E.A. degree (postgraduate diploma) in micro and nano electronics and the Ph.D. degree in microelectronics and with a dissertation on the enhancement of gas sensors selectivity by the use of noise spectroscopy from the University of Provence, Marseille, France, in 2002 and 2006, respectively. He was with the Laboratory of Materials and Microelectronics of Provence, Marseille, where he was involved in MEMS, gas sensors, and selectivity enhancement. In 2008, he joined the Department of Electrical Engineering, Micro Electro Thermal Systems Unit, ENIS, University of Sfax. His research interests are in the field of MEMS, gas sensors selectivity enhancement strategies, and wireless sensor network

Thierry Contaret was born in Saint Germain-en-Laye, France, in 1975. He received the Ph.D. degree from the University of Montpellier II, France, in 2003, with a dissertation on noise modeling and simulation in submicron MOS transistors. After his post-doctoral research on noise characterization of advanced MOSFET with the Institut de Microélectronique Electromagnétisme et Photonique, Grenoble, France, he joined the MicroSensors Group, Institut Matériaux, Microélectronique, Nanosciences de Provence, Aix-Marseille University, Marseille, France, in 2006. He is currently works on improving the selectivity of gas sensors by using noise spectroscopy.

Jean-Luc Seguin was born in France in 1958. He received the Ph.D. degree from the University of Aix-Marseille II in 1983 with a dissertation on adsorption and wetting on graphite and the Habilitation degree from the University of Aix-Marseille III in 2000. He specializes in thin films preparation and characterization for applications in microsystems. Since 1997, he has been interested in gas microsensors and has developed a selective ammonia sensor based on $\mathrm{CuBr}$ mixed ionic conductor. $\mathrm{He}$ is currently a Professor with the Institute of Materials Microelectronic Nanosciences of Provence, Aix-Marseille University, Marseille, France, working on gas sensors and selectivity enhancement strategies, including the adsorption-desorption noise spectroscopy, low-noise amplifier design, and ultra-low power and wireless systems. 